\title{
Embedded DSP-based Telehealth Radar System for Remote In-door Fall Detection
}

\author{
Carmine Garripoli, Marco Mercuri, Student Member, IEEE, Peter Karsmakers, Ping Jack Soh, Student \\ Member, IEEE, Giovanni Crupi, Senior Member, IEEE, Guy A. E. Vandenbosch, Fellow, IEEE, \\ Calogero Pace, Member, IEEE, Paul Leroux, Senior Member, IEEE, and Dominique Schreurs,
} Fellow, IEEE

\begin{abstract}
Telehealth systems and applications are extensively investigated nowadays to enhance the quality-of-care and, in particular, to detect emergency situations and to monitor the well-being of elderly people, allowing them to stay at home independently as long as possible. In this paper, an embedded telehealth system for continuous, automatic, and remote monitoring of real-time fall emergencies is presented and discussed. The system, consisting of a radar sensor and base station, represents a cost-effective and efficient healthcare solution. The implementation of the fall detection data processing technique, based on the Least-Square Support Vector Machines (LS-SVM), through a digital signal processor (DSP) and the management of the communication between radar sensor and base station are detailed. Experimental tests, for a total of 65 mimicked fall incidents, recorded with 16 human subjects (14 men and 2 women) that have been monitored for 320 minutes, have been used to validate the proposed system under real circumstances. The subjects' weight is between 55 and $90 \mathrm{~kg}$ with heights between 1.65 and $1.82 \mathrm{~m}$, while their age is between 25 and 39 year. The experimental results have shown a sensitivity to detect the fall events in real-time of $100 \%$ without reporting false positives. The tests have been performed in an area where the radar's operation was not limited by practical situations, namely signal power, coverage of the antennas, and presence of obstacles between the subject and the antennas.
\end{abstract}

Index Terms-Contactless, DSP platform, fall detection, health monitoring, LS-SVM, movement classification, radar remote sensing, telehealth systems, Zigbee communication.

Manuscript received March 31, 2014. This work was supported by FWO-Flanders, KU Leuven GOA Project, and the Hercules Foundation.

Carmine Garripoli is with the Department of Electrical Engineering, KU Leuven, 3001 Leuven, Belgium, and also with the Dipartimento di Informatica, Modellistica, Elettronica e Sistemistica, Università della Calabria, 87036 Rende (CS), Italy (e-mail: garripoli.carmine@gmail.com).

M. Mercuri, P. Karsmakers, G. A. E. Vandenbosch, P. Leroux, and D. Schreurs are with the Department of Electrical Engineering, KU Leuven, 3001 Leuven, Belgium (e-mail: Marco.Mercuri@esat.kuleuven.be; Peter.karsmakers@esat.kuleuven.be; Guy.Vandenbosch@esat.kuleuven.be; Paul.Leroux@esat.kuleuven.be; Dominique.Schreurs@esat.kuleuven.be).

P. J. Soh is with the Department of Electrical Engineering, KU Leuven, 3001 Leuven, Belgium, and also with the School of Computer and Communication Engineering, Universiti Malaysia Perlis, 02600 Arau, Perlis, Malaysia (e-mail: pingjack.soh@esat.kuleuven.be).

G. Crupi is with the Dipartimento di Ingneria Civile, Informatica, Edile, Ambientale e Matematica Applicata, University of Messina, 98166 Messina, Italy (e-mail: crupig@unime.it).

C. Pace is with the Dipartimento di Informatica, Modellistica, Elettronica e Sistemistica, Università della Calabria, 87036 Rende (CS), Italy (e-mail: calogero.pace@unical.it).

\section{INTRODUCTION}

$\mathrm{T}$ HE elderly population has been steadily increasing worldwide [1]. This situation, together with the shortage of nursing homes and the natural desire to stay at home, has resulted in a growing need for healthcare solutions to improve the quality of life for senior citizens and to increase the efficiency of systems for health and social care. Elderly people who live alone are usually exposed to health risks which in some cases may cause fatality. Moreover, in addition to chronic health problems, fall incidents are considered one of the major problems among the elderly worldwide. They often result in serious physical and psychological consequences [2]. The rapid detection of a fall event can reduce the mortality risk and increases the chance to survive the incident and return to independent living [3], [4]. For that reason, it is imperative to detect falls as soon as they occur such that immediate assistance may be provided.

Current fall detection systems are based on a necklace or wristwatch with a button that is activated by the patient in case of an accident. Other devices involve accelerometers and gyroscopes attached to the body [5], [6]. However, in emergency situations, this imposes an important risk factor. In fact, the person may forget to wear the device, or likely may no longer be able to press the button. Moreover, these devices produce discomfort and false alarms. An academic investigation of an accelerometer-based fall detector system using a biocompatible and impermeable skin patch has been reported in [7]. It can be carried by the user with the added value that the subject does not have to remember to wear it. The ideal solution is therefore a contactless approach that avoids the need for actions by the elderly person. Systems under investigation in the latter category are based on video cameras [8]-[11], floor vibration [12], and acoustic sensors [13]. In the case of the video camera method, researchers are currently trying to address challenges related to low light, field of view, and image processing, but also privacy is a concern [8]-[11]. Floor vibration and acoustic sensors have limited success due to the environmental interference and background noise [12], [13].

A novel and complementary investigation was presented by the authors in [14]-[16]. The resulted system, combining radar, wireless communication, and data-processing techniques, was 


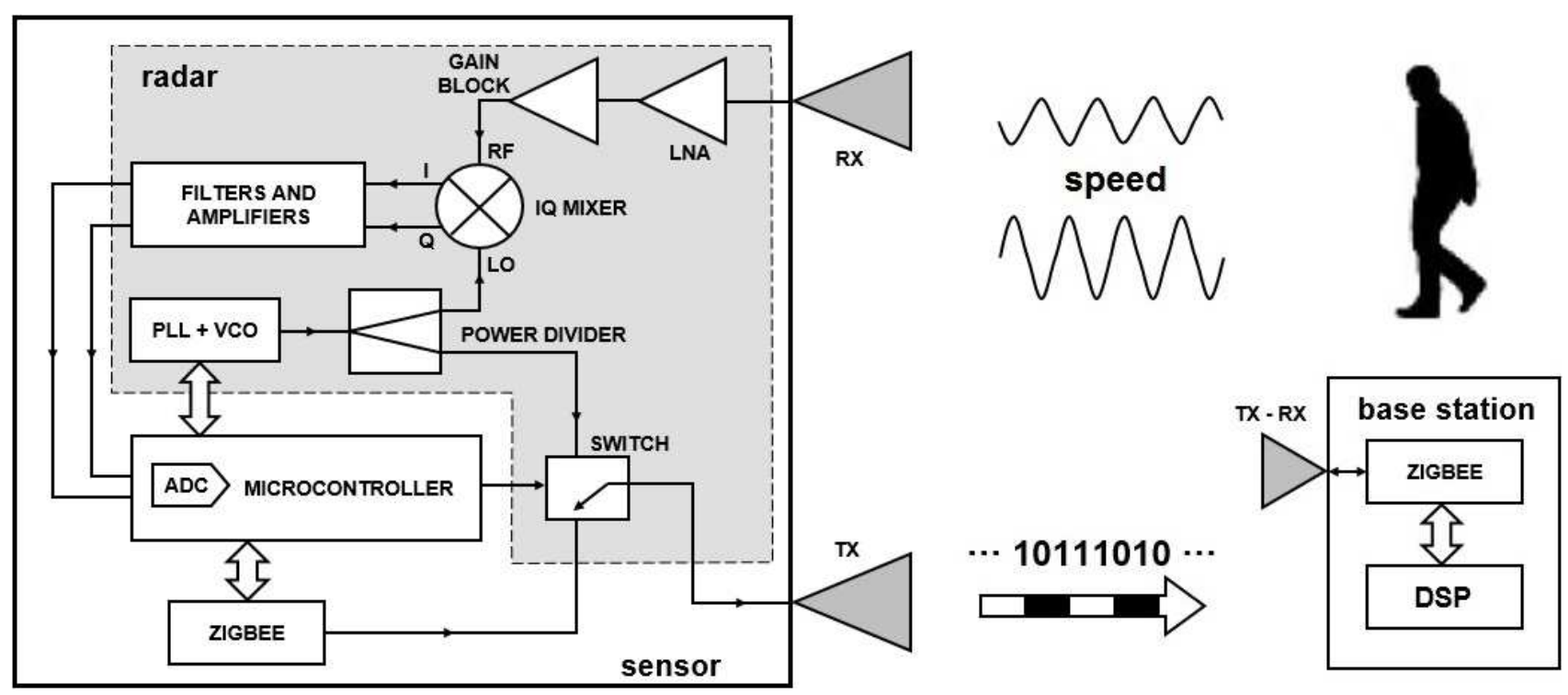

Fig. 1. Block diagram of the fall detection telehealth system.

demonstrated in an in-door environment to detect fall emergencies and to localize persons without the need of radio-frequency identification (RFID) tags attached to the person [16]. The system consists of a radar sensor that detects the monitoring signals of a person and transmits this information to a base station for remote data processing. The described base station consists of a Zigbee module, a microcontroller, and laptop, resulting in a non-compact and energy-inefficient solution. Moreover, the system was not able to work in real-time. In fact, the speed signals, consisting of one single activity (i.e., falling, walking) with known starting and end time points, were collected, stored, and processed offline later.

In comparison to the previous work [16], a cost-effective and energy-efficient base station based on a DSP embedded platform has been developed to detect fall emergencies in real-time. The implementation of the data processing technique to operate in real-time, the management of the communication between radar sensor and base station, together with design challenges, practical limitations, and their solutions, are presented and discussed.

Other interesting works on contactless fall detection using radar techniques were reported also in [17]-[19]. In these papers, a sequence of feature vectors, as a result of the STFT (Short Time Fourier Transformer), is transformed to a single feature vector of fixed length per segment. Hence, each activity can be represented by a single feature vector which can be the basis for standard machine learning methods such as Least Squares Support Vector Machines (LS SVM). However, in this proposed work an activity is represented by a sequence of vectors (with possibly different lengths). Within the LS-SVM framework it is feasible to work on such data by choosing a specific kernel function that is suited to process varying-length sequences of vectors. For this purpose, the Global Alignment (GA) kernel [20] was used in this work.
More specifically, the sequences of vectors are computed using the STFT. Therefore, each vector containing the spectral information of a single time window, considers a radar signal segment with a movement activity at some position (beginning, middle, end). The use of the GA kernel allows to correctly compare two segments with the, to be detected, movement positioned differently. This without relying on some energy selection criterion that select small parts of the segment to construct a fixed length vector but by comparing the frequency signatures. The details of our classification strategy are described in [15]. Moreover, in [17]-[19] the radar data were collected and processed offline, and nothing was mentioned about full radar-based telehealth system, where the monitoring signals are transmitted wirelessly to a base station unit to process them in real-time.

The telehealth monitoring system is introduced in Section II. The implementation of the data processing technique by means of a DSP platform is detailed in Section III, while the experimental results are described in Section IV.

\section{FALl DeteCtion TeleheAlth SyStEM}

In this section, the telehealth system described in [16], is briefly introduced to better understand the novelty of this work which will be detailed in the next section. However, as opposed to [16], where the base station consists of a Zigbee module, a microcontroller, and a laptop, a DSP-based base station has been developed and used in this work.

The telehealth system consists of a sensor, combining radar, computational, and wireless communication capabilities, and a base station for data processing (Fig.1). A Continuous Wave (CW) waveform at $5.8 \mathrm{GHz}$ is generated and transmitted toward a human target to detect its speed produced during daily activities, such as falling, walking, random movements. In fact, by the Doppler effect, a radio wave reflected by a moving target undergoes a frequency shift proportional to its 
velocity. The reflected echo, containing the person's speed information, is collected by the receiver. The resulting baseband signals are digitized and transmitted to the base station to distinguish fall events from normal movements.

The sensor architecture and the DSP-based base station are described in Subsection II.A and in Subsection II.B, respectively. Moreover, the technique to distinguish fall events from normal movements is described in Subsection II.C.

\section{A. Sensor Architecture}

The radar sensor is composed of a radar module, a microcontroller, and a Zigbee module (Fig. 2). It also mounts a two-element bow-tie antenna to support both the wireless communication between the sensor and the base station and the radar working frequency. The antenna was optimized to reduce the backscattering and crosstalk effects, presenting also a semispherical radiation pattern to cover a whole room [21].

The radar module integrates a Fractional-N Phase-Locked Loop (PLL), a power divider, a radio frequency (RF) switch, a low noise amplifier (LNA), a gain block, an In-Phase and Quadrature (IQ) mixer, baseband filters and amplifiers.

The $5.8 \mathrm{GHz}$ single tone is generated by the PLL that is configured by the microcontroller. This signal is sent to the power divider that splits it in two branches. The first output is connected to the RF switch. The latter is controlled by the microcontroller to alternatively connect the radar transmitter and the Zigbee module to the transmitter antenna. The signal reflected from the target is received, amplified, and then mixed with a copy of the transmitting signal. On the receiving path, the signal is amplified by the LNA and the gain block, for a total gain of $30 \mathrm{~dB}$. The output of the gain stage is connected to the RF input of the mixer. The local oscillator (LO) input of the mixer is connected to the second output of the power divider. The IQ baseband signals produced by the mixer are amplified, filtered, and adapted to the ADC's dynamic range. The 10-bit ADC is integrated into the microcontroller and works with a sampling frequency of 250 $\mathrm{Hz}$, such that every $4 \mathrm{~ms}$ an IQ sample pair is acquired and digitized. Since the Zigbee module transmits only frames organized in bytes, each IQ sample pair is mapped in 3 bytes. These samples are packed in a frame of 75 bytes and then transmitted wirelessly to the base station through the Zigbee module every $100 \mathrm{~ms}$. This transmission requires about $3 \mathrm{~ms}$ and is performed in between sampling instants. This guarantees both that the sampling rate is always constant and that the person is continuously monitored.

\section{B. DSP-based Base Station Architecture}

The developed base station consists of a Zigbee module and the TMS320C6678 DSP platform (Fig. 3). This processor implements an SIMD (Single Instruction, Multiple Data) approach in floating point instructions in single (32 bits) and double (64 bits) precision, allowing to maintain the same data format of Matlab scripts. It integrates 8 DSP cores that run at 1 $\mathrm{GHz}$ each, and it has shared integrated static random access memory (SRAM) of $4 \mathrm{MB}$. It also has a high speed external memory controller, which supports DDR3 up to $1600 \mathrm{MT} / \mathrm{s}$,

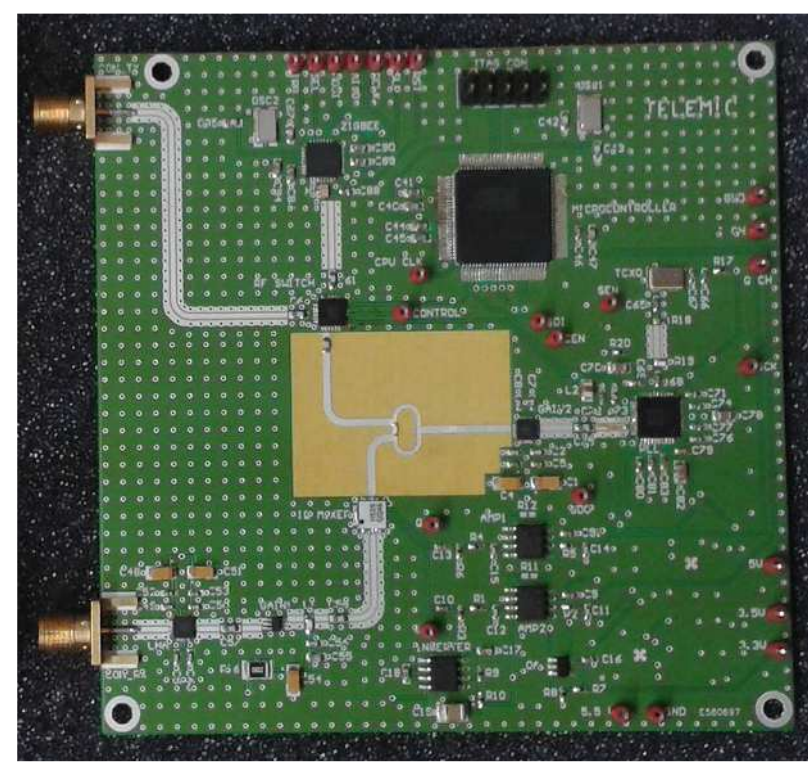

Fig. 2. Developed radar sensor.

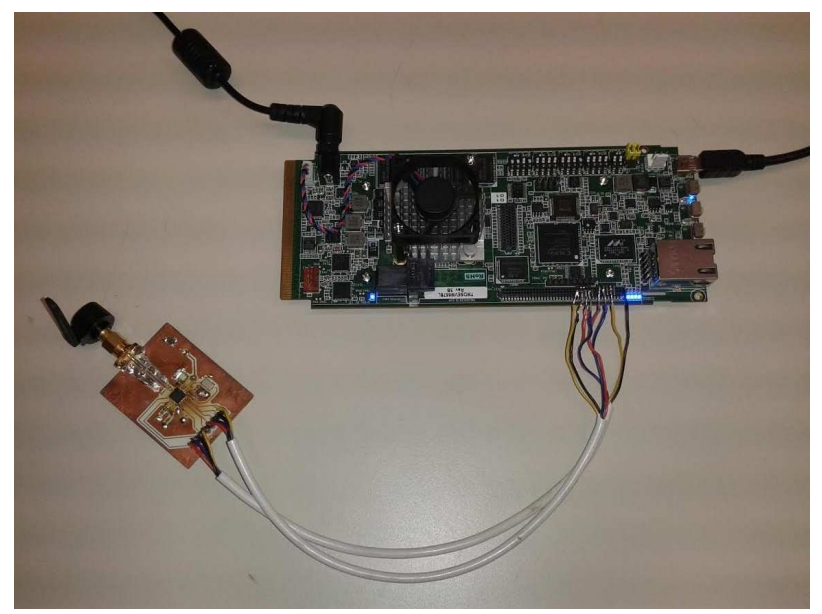

Fig. 3. DSP-based base station.

together with a data bus width of 64 bits that is capable of 12.8 GB/s. For this particular DSP, mathematic and signal processing hand assembly optimized libraries are also available, to speed up the process of optimizing signal processing algorithms.

The DSP is connected through a Serial Peripheral Interface (SPI) to the Zigbee IC. The latter acts as a slave in the SPI interface while the DSP is the master. There are also 3 control lines, namely RST (active low reset), SLP (sleep), and IRQ (Interrupt ReQuest). The latter is the interrupt pin that is set every time a complete frame is received. This pin in connected to a DSP GPIO (General-Purpose Input/Output) port that triggers the routine for receiving the transmitted frame from the Zigbee module.

\section{Data Processing Technique}

In this Subsection the technique to distinguish fall events from normal movements, which was already explained in [16], is briefly introduced for completeness to understand how the 
system has been extended to work in real-time. In [16], in fact, the speed signals, consisting of one single activity with known starting and end time points, were collected, stored, and processed offline later. Moreover, in [16] the validation was performed considering only falling and walking movements.

The digitized speed baseband signals are processed offline in Matlab. A movement classification based on a LS-SVM approach combined with a GA kernel [15] is applied to analyze the digitized baseband speed signals in order to distinguish fall events from the other activities. The fall detector aims at assessing the changes in speed experienced during a fall or a normal movement. The developed algorithm consists of two stages of data analysis, namely the training phase and the testing phase.

The training phase consists of event detection and segmentation, preprocessing, and model estimation. For each collected signal, consisting of one single activity, the energy's peak is first detected and then the signal is cut around it to create a segment of 2 seconds, considered sufficient to cover the details of the activities and mainly of a fall event. Those segments are used to build a data set. After that, the data is preprocessed (or standardized) such that each dimension has zero mean and unit standard deviation. Next it is transformed using the STFT from which only the magnitude spectrum is retained. The resulting data is standardized again and the learning process is started. The model is then created and stored in a memory to be used in the testing stage. In the model, the activities are divided in two groups, namely fall events and normal movements.

In order to validate it, an independent test set, with data not used in the training phase, is needed. It is built, acquiring test signals, consisting of one activity, that undergone the same operations of event detection, segmentation, and preprocessing described for the training phase. The resulting test set is then compared to the model.

\section{FALL DETECTOR}

In this Section the implementation of the data processing technique to operate in real-time is detailed. In particular, the synchronization and the communication between radar sensor and base station are explained in Subsection III.A. The training phase and the testing phase described in Subsection III.B and in Subsection III.C, respectively. The classification implementation is detailed in Subsection III.D.

\section{A. Sensor-Base Station Communication}

In order to synchronize the system, the Zigbee modules of the sensor node and of the base station are set at power-on as receiver and transmitter, respectively. The sensor node will stay in this modality until it receives a frame of two fixed bytes (coins) from the base station, meaning that it is ready to process the monitoring signals. The base station sends the coins to the sensor. The latter checks whether the received bytes are equal to a copy of the coins saved in its memory. If that condition is verified, the sensor is changed immediately to transmission mode and it sends back the received coins to the base station that in the meanwhile has set itself as receiver. Once they have been received, the base station checks whether the received coins are equals to the initial token sent at the beginning of the synchronization process. In the case this procedure is correct, a message, meaning that the synchronization has been achieved, is printed out to the debug monitor. On the other hand, if this procedure is not correct or the coins are not received within one second, an error message is printed out and the system must be rebooted to restart the

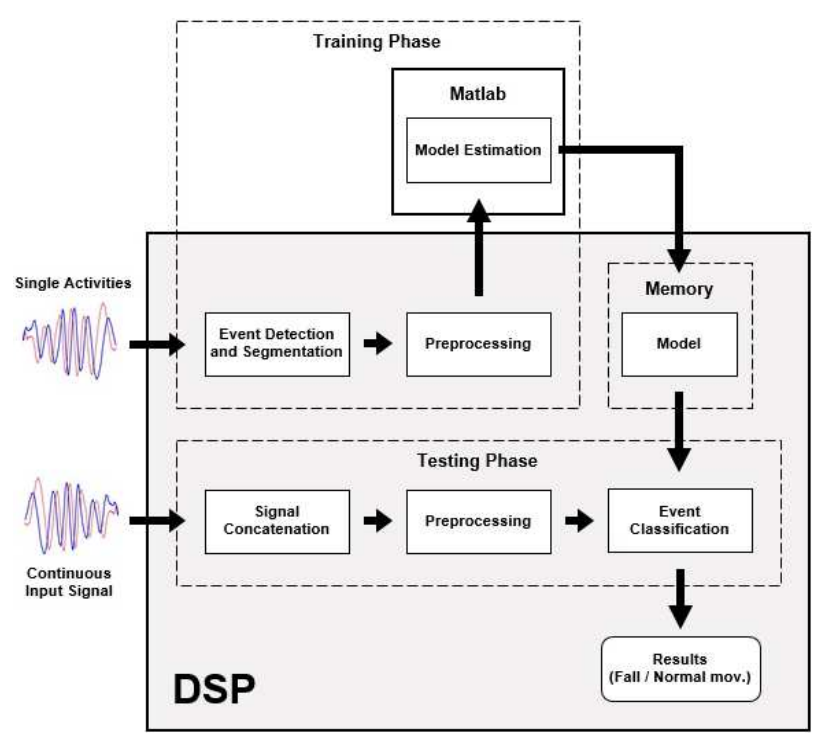

Fig. 4. Block diagram of the implemented LS-SVM with GA kernel technique.

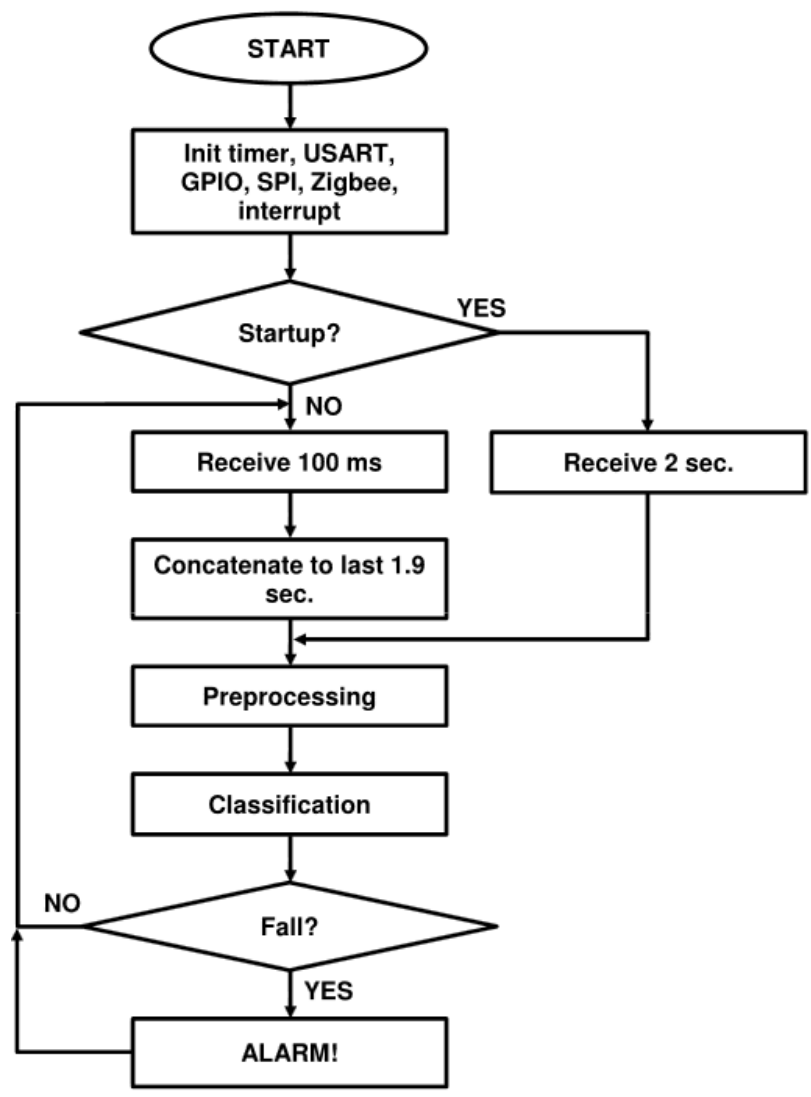

Fig. 5. Fall detection testing phase flow chart. 
synchronization process.

The Zigbee module in the base station receives the speed frames every $100 \mathrm{~ms}$. Every time a new frame is received, the IRQ pin of the Zigbee module is set. This triggers an interrupt to the DSP, such that the data can be read.

\section{B. Training Phase}

The simplified block diagram of the training phase is shown in Fig. 4. In order to create the model, a routine has been implemented to acquire the signal activities. In particular, it allows acquiring signals of 8 seconds. During this time a subject should perform only one activity (i.e., falling, walking, random movement). Moreover, in between successive acquisitions, there is a time slot of $30 \mathrm{sec}$. such that the person can prepare himself/herself to mimic another activity.

After the acquisition, the activity energy's peak of each signal is extracted such that the signal is cut around this peak to produce a segment of 2 seconds. All these segments are arranged in a matrix and the preprocessing is performed, where the STFT is involved, such that the segments are first cut into 50\% overlapping frames that are multiplied with a Hamming window, and then the Fast Fourier Transformer (FFT) is computed on each of these frames.

The result of the preprocessing is sent to a laptop for the model estimation, where an off-the-shelf algorithm implemented in a Matlab script was involved. The generated model is saved as a three-dimensional matrix in a $\mathrm{C}$ header file and is then stored in the internal SRAM of the DSP.

The operations of event detection, segmentation, and preprocessing could be directly run in Matlab. However, in order to perform the FFT, the DSP uses assembly optimized libraries that produces results slightly different from Matlab. The differences would have resulted in classification errors during the testing phase that is performed by the DSP.

\section{Testing Phase}

The testing phase consists of signal concatenation, preprocessing, and classification (Fig. 4). In order to process a continuous stream of radar signals consisting of multiple activities invoked at unknown instants, a signal concatenation technique has been investigated in the testing phase. More precisely, every time a new frame is available (i.e., $100 \mathrm{~ms}$ of new speed signals), the relative I/Q samples are concatenated with the last $1.9 \mathrm{sec}$. of the previous signals to create a segment of $2 \mathrm{sec}$., that is preprocessed and classified. The system manages also the power-on situation. In fact, when it is powered on, it accumulates $2 \mathrm{sec}$. of signals (i.e., 20 frames of $25 \mathrm{I} / \mathrm{Q}$ sample pairs). After this first segment is processed, every new frame will be concatenated to the previous 19 . This procedure involves an overlap of $95 \%$ among segments. This large overlap is used to improve the performance of the system. In fact, a larger overlap involves a higher number of classifications such that a fall event will be considered over multiple segments. This makes the system more immune to the noise that could generate a false positive in a single segment classification. An alarm is activated if a fall event has been detected. The flow chart of the testing phase is shown in Fig. 5.

\section{Parallelization Classification Technique}

A custom parallelization technique involving the 8 cores of the TMDS320C6678 DSP has been implemented to perform real-time classification. The strict requirement is that a segment classification must be performed before a new Zigbee communication occurs, that means in a time shorter that 100 ms. In this work a fall event is detected when 3 consecutive segments have been classified as a fall. This involves that the maximum time to detect a fall should be shorter than $400 \mathrm{~ms}$.

The pseudo-code of the classification is shown in Fig. 6. The core of the algorithm is to determine the Kernel array $K$, whose elements are calculated by the function computeGAK(Test, Training), that is an off-the-shelf C code that computes the Global Alignment Kernel for the LS-SVM algorithm [15], [20]. The $K$ array is generated starting from the Training matrix and the Test matrix, which are multidimensional arrays, resulting from the model estimation and the preprocessing in the testing phase respectively. The Training matrix, consisting of $N_{\text {train }}$ elements, contains the result of the preprocessing performed in the training phase. In particular, each activity is segmented into sequences of 2 seconds (i.e., 500 samples), on which the STFT is applied. Since the latter involves 32-sample windowing with $50 \%$ overlap, 30 sequences of 32 samples are generated. The meaningful information for the classification resides in the first 8 samples of the STFT, meaning that, for each activity, 30 non-redundant sequences of 8 samples are produced. These sequences are then organized into a two-dimensional (2-D) array of dimension $30 \times 8$. This means that the Training matrix consists of $N_{\text {train }}$ elements, where each element is a $30 \mathrm{x}$ 8 matrix. This makes the Training matrix a three-dimensional (3-D) array of dimension $N_{\text {train }} \times 30 \times 8$. Similar considerations are valid for the Test matrix. Since in the real-time implementation only a single 2-sec. segment of signal is classified each time, the resulting Test matrix is a 2-D array of dimension $30 \times 8$.

For each iteration of the loop (Fig. 6), 8 elements of the Kernel array $K$ are computed at the same time by the 8 cores of the DSP. Each core invokes the function computeGAK() independently. Finally, the function $f(K)$ returns a number that, compared to a threshold, determines whether the segment contains a fall or normal movement data.

In order to generate the $K$ array, a fork/join method, based on an Inter Processor Communication (IPC) technique, has been designed. It exploits the internal shared memory of an architecture to synchronize and exchange data among processing units. In doing this operation, a master/slave approach has been chosen, where core 0 is the master core that manages the fork/join processes and also computes Kernel elements. In particular, during the fork process, the arguments for the function computeGAK(Test, Training) are passed to the 8 cores, while in the join process, the results of each core are collected. More precisely, during the fork phase, the master core core 0 passes to each slave core both the address of one element of the Training matrix and the address of the Test matrix. Each core therefore compares each time the $j$-th $30 \times 8$ element of the Training matrix with the $30 \times 8$ Test matrix. 
The result of the comparison is a double precision float that is saved, during the join phase, into the Kernel array $K$ at the $j$-th position. This operation is possible because the elements of the Training matrix are independent from each other.

A Finite State Machine (FSM) has been designed to control the 7 slave cores (Fig. 7). It consists of two states, namely STATE0 and STATE1, and two flags, Status and Idle. Initially, all the slave cores are in STATE0, meaning that they are in idle mode, with Idle flag set to 1 . They remain in this state until the master sets the Status flag after having passed them the computeGAK() arguments. When this happens, the slave cores clear their Idle flags and go to STATE1. In this state, the slave cores call the function computeGAK(). When all the cores have cleared the Idle flag, the master core can also call the computeGAK() function. After the results are computed, each slave core sets the Idle flag and waits for Status flag to be cleared by the master core, meaning it has received the results. When this happens, the slave cores return to STATE0, waiting for new data to be processed.

In order to exchange arguments and to control the FSM properly, 8 shared memory messages have been designed for the 8 cores. Each message has the format of a $\mathrm{C}$ structure as shown in Fig. 8, where:

- status is the control flag that is used to trigger the start of the slave core operation;

- idle is the flag that indicates whether a slave core is processing data or is waiting to receive new data (i.e., idle state);

- *seq 1 is the pointer to the $j$-th $30 \times 8$ element of the Training matrix that needs to be processed;

- $*_{s e q} 2$ is the pointer to the $30 \times 8$ Test matrix that needs to be processed;

- $n X$ is the first dimension of each element of the Training matrix (i.e., 30 in this work);

- $n Y$ is the first dimension of each element of the Test matrix (i.e., 30 in this work);

- dimvect is the second dimension of each element both of the Test matrix and of the Training matrix (i.e., 8 in this work);

- sigma and triangular are two parameters generated in the training phase of the classification. The values of these parameter are evaluated in order to minimize the cost function of the LS SVM formulation [15], [20];

- return_value is the result of the comparison between one element of the Test matrix and one element of the Training matrix.

When all the elements of the Kernel matrix $K$ are calculated, the master core calculates the values $f(K)$ and establishes whether the event is a fall or a normal movement.

The implemented parallelization technique together with the signal reception, managed by interrupts, represents a flexible solution even if multiple sensors are used. In fact, the DSP is able to receive frames from the Zigbee module even if the classification is running, meaning that a sensor can transmit data at any time, without requiring a complex synchronization of the whole network. In fact, the interrupt service routine (ISR) is served only by the master core, that reads and stores

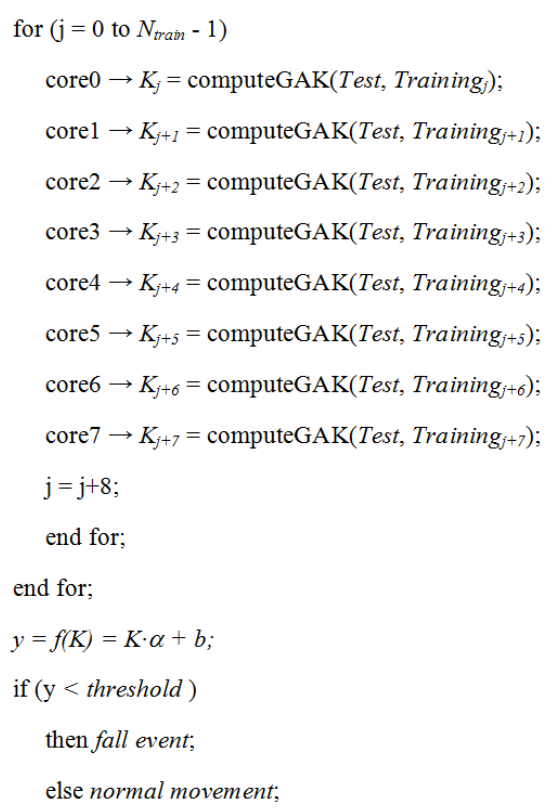

Fig. 6. Classification pseudo-code. $N_{\text {train }}$ represents the number of matrices in the Training structure. The vector $\alpha$ and the constant $b$ are variables estimated in the training phase.

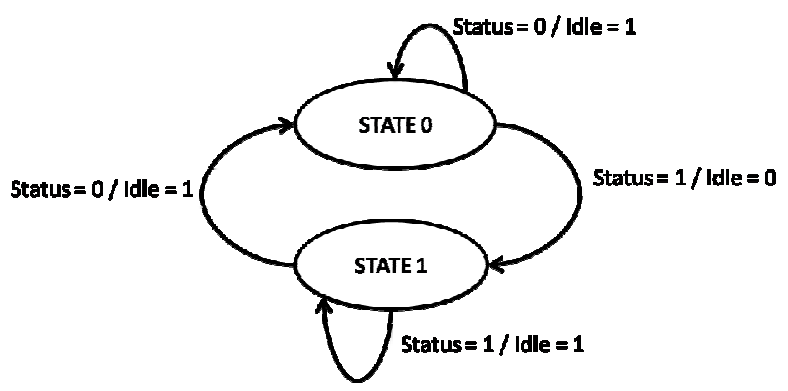

Fig. 7. FSM of the slave cores.

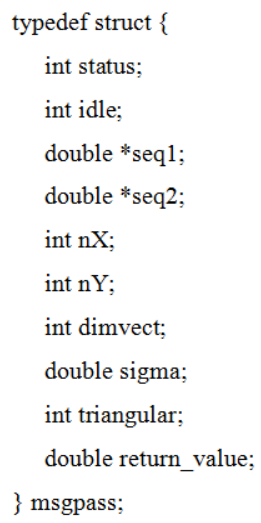

Fig. 8. Example of message used in the Shared Memory IPC technique.

the new data in a buffer, while the slave cores can continue to perform their operation without interruption. This means that the maximum number of sensors that can be managed by the base station is limited only by the number of classifications that can be performed within $100 \mathrm{~ms}$. 


\section{EXPERIMENTAL RESULTS}

Experimental tests have been performed with human volunteers in a room of $5 \times 5 \mathrm{~m}^{2}$ (Fig. 9). Furniture, a metal shelf, tables, a sofa, PCs, and chairs were positioned to mimic a real room setting. The sensor was fixed to the wall at a height of $1.25 \mathrm{~m}$ while the base station was positioned on a desk about $4 \mathrm{~m}$ away the sensor.

The classification model has been created on signals acquired on three volunteers in different positions in the room who did not participate in the testing phase. It includes 40 random walking activities, 30 activities of sitting down and standing up, 40 fall activities, considering both hard falls, where the person falls directly to the ground, and soft falls, by which the person tries to avoid the incident by grabbing objects. In addition, 20 random movements, such as opening the window, moving a chair, have been also considered.

In order to validate the real-time fall detector, 64 tests, for a total of 65 simulated fall events, have been performed on 16 volunteers ( 14 men and 2 women) that have been continuously monitored for five minutes each. This means that this validation considered 320 minutes of measurements. Therefore, considering that a segment of signal is processed every $100 \mathrm{~ms}$ and also the power-on condition (namely the system should first accumulate $2 \mathrm{~s}$ of signals), this validation has been performed over 190784 testing segments of radar signal. The subjects were allowed to move without restrictions within the antenna's beamwidth, meaning that they could mimic all the typical movements that are normally achieved in a domestic environment (i.e., walking, talking at the cellular while walking in the room, dropping object or even a chair, walking with a cane and with a walker, sweeping, working at the pc, watching films, resting on the sofa, eating, open windows, drinking water, etc.). One single volunteer was present in the room at a time and performed only one fall during the monitored period. Only in one test, the volunteer was invited to mimic two consecutive falls where the subject experiences a first fall, then tries to gets up, and then falls

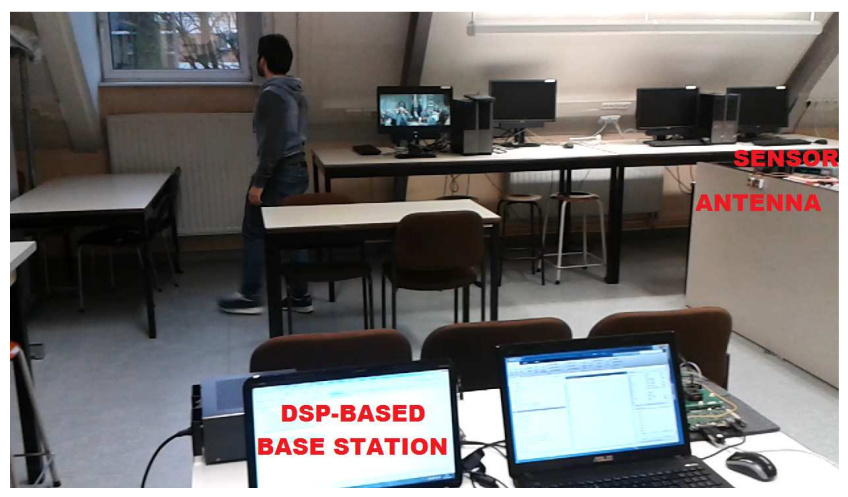

Fig. 9. Real room environment.

again. The mimicked fall incidents included both hard falls and soft falls. Other simulations consider situations where the person falls from a chair, loses the equilibrium while walking with a cane or with a walker, sits and misses the chair, loses the equilibrium and falls on a chair, is bent over and tries to get up. To this end, videos recorded in nursery homes with real fall incidents have been watched in order to mimic real life situations. The subjects' weight is between 55 and $90 \mathrm{~kg}$ with heights between 1.65 and $1.82 \mathrm{~m}$, while their age is between 25 and 39. The experimental results have shown a sensitivity in detecting the mimicked fall events in real-time of $100 \%$. Moreover, no false positives have been reported.

It should be noted that this validation considers only falls where the volunteers are located at angles between 0 and about 45 degrees from the line of sight (LoS) of the antenna, otherwise the related radial speeds would produce lower Doppler frequencies such that fall incidents will be classified as normal movements. Other possible limitations of the radar system are when the person is in a position outside of the antenna beamwidth and when his/her reflection is obstructed by furniture. Also the target absolute distance could represent a limitation. In fact, the longer the distance is, the weaker is

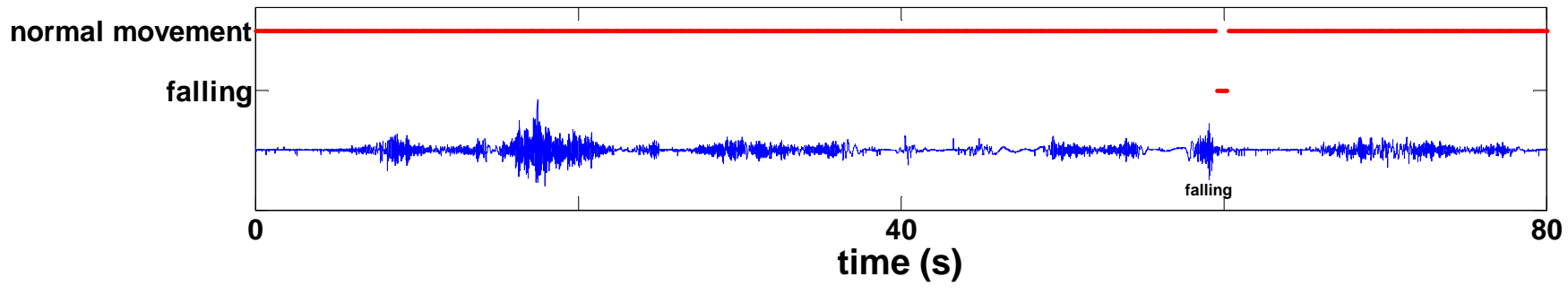

(a)

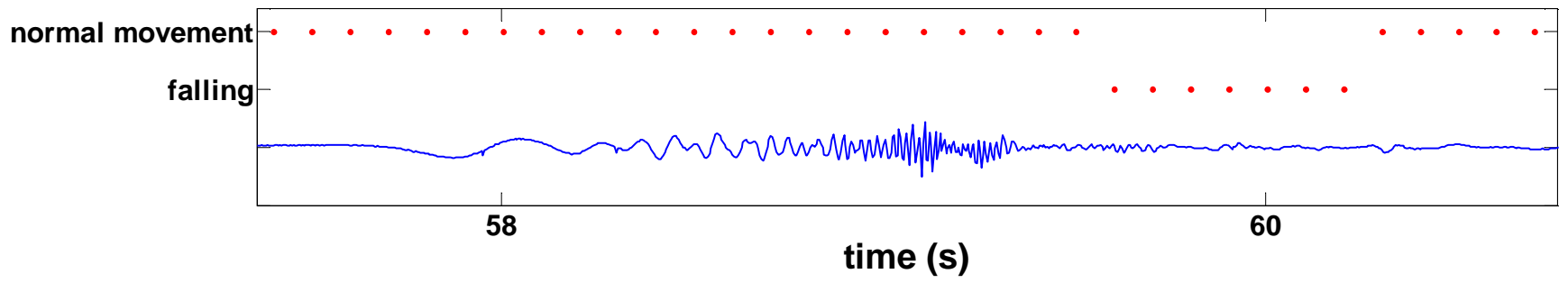

(b)

Fig. 10. Classification results (a) of a signal containing multiple activities and (b) zoom of the related fall event. In this example, the results of the classification 


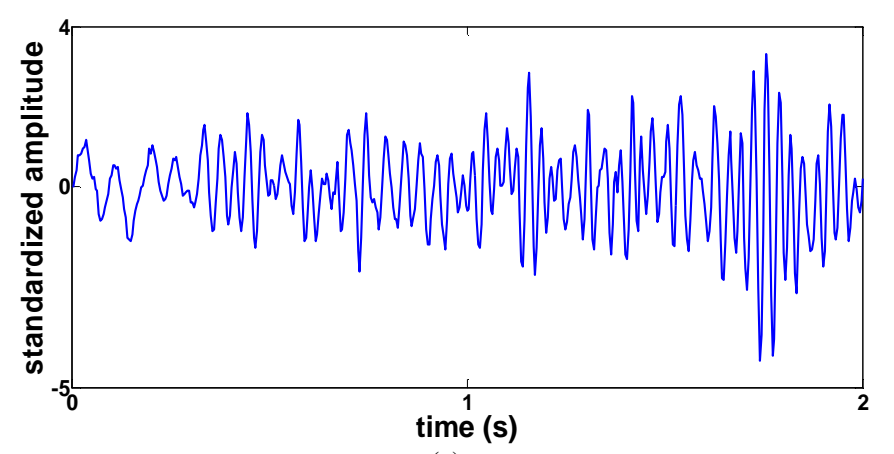

(a)

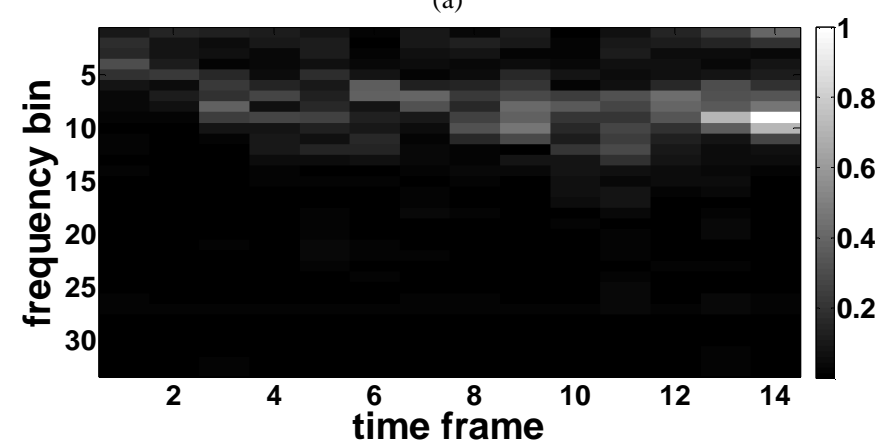

(c)

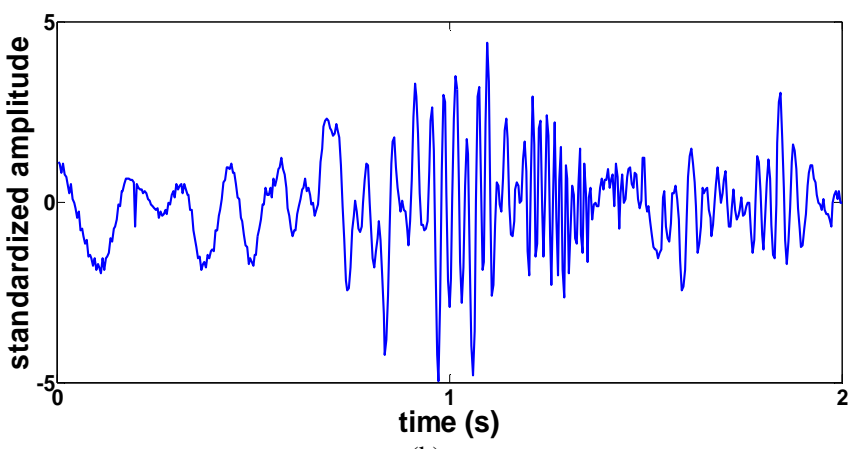

(b)

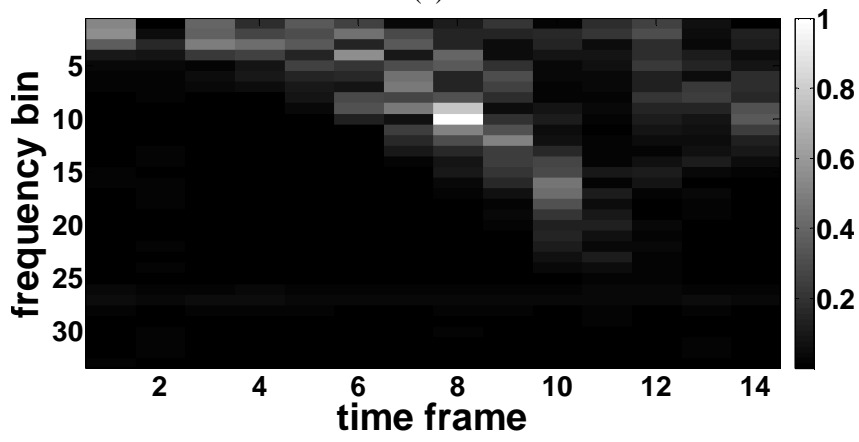

(d)

Fig. 11. Speed signal during (a) a walking movement and during (b) a fall event. Spectrograms corresponding (c) to the walking movement and (d) to the fall event.

the target's reflection, such that it may no longer lie within the radar dynamic range and then it will be buried in the noise. This problem can be mitigated both by increasing the output power and by increasing the receiver's gain. However, the maximum transmitted power is limited by the spectral masks of the standards (i.e., maximum allowable power in in-door environments), while the receiver's gain is limited by the unwanted reflections generated by the radar itself (i.e., crosstalk, backscattering, isolation between transmitter and receiver). However, the radar dynamic range does not represent a serious problem in a typical room of $5 \times 5 \mathrm{~m}^{2}$. Obviously, these limitations could be avoided by using multiple sensors.

Fig. 10 shows the classification results on a small portion of a signal containing multiple random activities and a fall event invoked at about 58 seconds. Each dot represents the class where a segment of $2 \mathrm{sec}$. of signal has been assigned. The event was classified as fall for seven consecutive segments.

The measured time to process a segment of $2 \mathrm{sec}$. of signal is about $16 \mathrm{~ms}$. This means that, considering Zigbee transmissions every $100 \mathrm{~ms}$, with the developed approach, it is possible to process signals coming from six sensors without loss of information. Since an alarm is activated when three consecutive segments are classified as a fall, the maximum time to detect the incident is about $316 \mathrm{~ms}$.

Figures 11.a, 11.b represent the resulting speed signals of a walking movement and of a fall event, respectively. The frequency of the signals is proportional to the radial velocity of the person during the movement. Figs. 11.c, 11.d show the two spectrograms corresponding to the movement activities of
Figs. 11.a, 11.b, respectively. The horizontal axis represents time, the vertical axis frequency, a third dimension indicating the amplitude of a particular frequency at a particular time is represented by different shades of gray. In this example a sliding window size of 64 samples with $50 \%$ overlap is adopted. In case of a fall an increase in dominant frequency over time is observed while for the walking activity the dominant frequency per time window remain within a small specific band.

\section{CONCLUSION}

In this paper, a telehealth system aiming at remote fall detection in an in-door environment has been presented. It consists of a microwave radar sensor and a wirelessly connected base station for data processing. The implementation of the fall detection algorithm by means of a DSP platform has been presented. Experimental results conducted with human subjects under real circumstances have shown a sensitivity to detect fall events in real-time of $100 \%$, without reporting any false positives, with a maximum delay of about $316 \mathrm{~ms}$. The tests have been performed in an area where the radar's operation was not limited by practical situations, namely signal power, coverage of the antennas, and presence of obstacles between the subject and the antennas. Moreover, the proposed approach would allow to process at the same time up to six sensors without loss of information. This system is the result of the convergence of information, wireless technologies, and radar techniques, and is in-line with the growing need for health technologies and applications to 
enhance the quality-of-care for elderly people both in home and clinical environment. Next step is to integrate multiple sensors in a wireless sensor network to detect fall incidents in all the directions and to perform in-door positioning by implementing a trilateration technique.

\section{REFERENCES}

[1] UN World Population Prospects: The 2012 Revision, http://esa.un.org/unpd/wpp/index.htm, Population Division of the Department of Economic and Social Affairs of the United Nations Secretariat, 2013.

[2] C. J. L. Murray and A. D. Lopez, "Global and regional descriptive epidemiology of disability: Incidence, prevalence, health expectancies and years lived with disability," Global Burden Dis., vol. 1, pp. 201-246, 1996

[3] E. Dejaeger, S. Boonen, J. Coussement, and K. Milisen, "Recurrent falling, osteoporosis and sarcopenia, three major problems, an integrated approach," J. Gerontol Geriatr, vol. 40, no. 6, pp. 262-269, Dec. 2009.

[4] S. R. Lord, C. Sherrington, and H. B. Menz, Falls in Older People: Risk Factors and Strategies for Prevention. Cambridge, U.K: Cambridge Univ., 2007.

[5] T. Zhang et al., "Fall detection by embedding an accelerometer in cellphone and using KFD algorithm," Int. J. Computer Science Network Security, vol. 6, no. 10, pp. 227-284, Oct. 2006.

[6] A. K. Bourke, and G. M. Lyons, "A threshold-based detection algorithm using a bi-axial gyroscope sensor," Med. Eng. Phys., vol. 30, no. 1, pp. 84-90, Jan. 2006.

[7] D. Naranjo-Hernandez, L. M. Roa, J. Reina-Tosina, and M. A. Estudillo-Valderrama, "Personalization and Adaptation to the Medium and Context in a Fall Detection System," IEEE Trans. Inform. Technol. Biomed., vol. 16, no. 2, pp. 264-271, March 2012.

[8] T. Lee and A. Mihailidis, "An intelligent emergency response system: preliminary development and testing of automated fall detection," $J$. Telemed Telecare, vol. 11, no. 4, pp. 194-198, 2005.

[9] F. Wang, M. Skubic, C. Abbott, J. M. Keller, "Body Sway Measurement for Fall Risk Assessment Using Inexpensive Webcams," in Proc. 32nd Annu. Int. Conf. IEEE Eng. Med. Biol. Soc., Buenos Aires, Argentina, Aug. 31-Sept. 4, 2010, pp. 252-255.

[10] A. Edgcomb, and F. Vahid, "Automated Fall Detection on Privacy-Enhanced Video," in Proc. 34th Annu. Int. Conf. IEEE Eng. Med. Biol. Soc., San Diego, California, USA, Aug. 28-Sept. 1, 2012, pp. 252-255.

[11] E. Auvinet, F. Multon, A. Saint-Arnaud, J. Rousseau, and J. Meunier, "Fall Detection With Multiple Cameras: An Occlusion-Resistant Method Based on 3-D Silhouette Vertical Distribution," IEEE Trans. Inform. Technol. Biomed., vol. 15, no. 2, pp. 290-300, March 2011.

[12] Y. Zigel, D. Litvak, and I. Gannot, "A method for automatic fall detection of elderly people using floor vibrations and sound - Proof of concept on human mimicking doll falls," IEEE Trans. Biomedical Eng., vol. 56, no. 12, pp. 2858-2867, Dec. 2009.

[13] L. Yun, Z. Zhiling, M. Popescu, and K.C. Ho, "Acoustic fall detection using a circular microphone array," in Proc. Аnпи. Int. Conf. IEEE Eng. Med. Biol. Soc., Sept. 4, 2010, pp. 2242-2245.

[14] M. Mercuri, D. Schreurs, and P. Leroux, "SFCW microwave radar for in-door fall detection," in Proc. IEEE Topical Conf. Biomedical Wireless Technol., Santa Clara, CA, USA, Jan. 15-18, 2012, pp. 53-56,

[15] P. Karsmakers, T. Croonenborghs, M. Mercuri, D. Schreurs, and P. Leroux, "Automatic in-door fall detection based on microwave rada measurements," in Proc. Eur. Radar Conf., Amsterdam, The Netherlands, Oct. 31-Nov. 2, 2012, pp. 202-205.

[16] M. Mercuri, P. J. Soh, G. Pandey, P. Karsmakers, G. A. E. Vandenbosch, P. Leroux, and D. Schreurs, "Analysis of an indoor biomedical radar-based system for health monitoring," IEEE Trans. Microwave Theory Tech., vol. 61, no. 5, pp. 2061-2068, May 2013.

[17] L. Liu, M. Poposcu, M. Skubic, M. Rantz, T. Yardibi, and P. Cuddihy, "Automatic Fall Detection Based on Doppler Radar Motion Signature," in Proc. 5th Int. Conf. Perv. Comp. Technol. Healthcare, Dublin, Ireland, May 23-26, 2011, pp. 222-225.

[18] L. Liu, M. Poposcu, M. Rantz, and M. Skubic, "Fall Detection using Doppler Radar and Classifier Fusion," in Proc. IEEE-EMBS Int. Conf.
Biom. Health Inform., Hong Kong and Shenzhen, China, Jan 2-7, 2012, pp. $180-183$.

[19] L. Liu, M. Poposcu, K. C. Ho, M. Skubic, and M. Rantz, "Doppler Radar Sensor Positioning in a Fall Detection System," in Proc. 34th Annu. Int. Conf. IEEE Eng. Med. Biol. Soc., San Diego, California, USA, Aug. 28-Sept. 1, 2012, pp. 256-259.

[20] M. Cuturi, "Fast global alignment kernels," in Proceedings of the 28th International Conference on Machine Learning (ICML-11), 2011, pp. 929-936.

[21] P. J. Soh, M. Mercuri, G. A. E. Vandenbosch, D. Schreurs, "A dual-band unidirectional planar bowtie monopole for a compact fall-detection radar and medical telemetry system," IEEE Antennas and Wireless Propagation Letters , vol. 11, pp. 1698-1701, Nov. 2012.

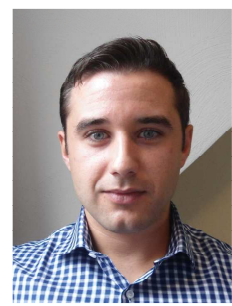

Carmine Garripoli was born in Locri, Italy in 1989. $\mathrm{He}$ received the Bachelor and Master degrees in electronic engineering from the Università della Calabria (UNICAL), Arcavacata di Rende, Italy, in 2011 and 2014, respectively. He is currently working towards the Ph.D degree within the MsM research group of the Department of Electrical Engineering, TU Eindhoven, The Netherlands.

His research interests reside in mixed signal integrated circuit for front ends, organic TFT technology, and electronics on flexible substrate.

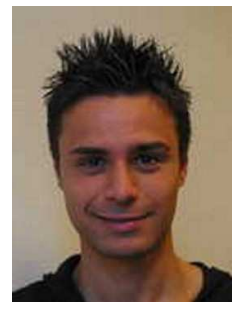

Marco Mercuri (S'12) was born in Lamezia Terme, Italy in 1985 . He received the Bachelor and Master degrees in electronic engineering from the Università della Calabria (UNICAL), Arcavacata di Rende, Italy, in 2006 and 2009, respectively. He is currently working towards the Ph.D degree within the TELEMIC research group of the Department of Electrical Engineering (ESAT), KU Leuven, Belgium.

His research interests include biomedical applications of microwave/RF, remote radar sensing, wireless sensors, and microwave/millimeter-wave measurements.

Mr. Mercuri is a student member of the IEEE Microwave Theory and Techniques (IEEE MTT-S) and the IEEE Engineering in Medicine and Biology Society. He was the recipient of the 2013 IEEE MTT-S Graduate Fellowship Award, and he obtained also the second place both to the 2013 IEEE President's Change the World Competition (PCW) and to the 2013 IEEE MTT-S YouTube/YouKu Video Competition.

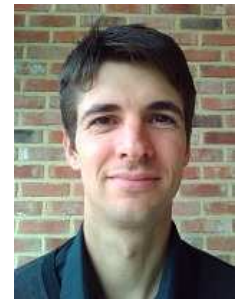

Peter Karsmakers was born on April 14, 1979. He received a M.Sc. degree in electronics-ICT engineering from the Katholieke Hogeschool Kempen (KHKempen), Belgium in 2001. In 2004 he received the degree of Master in Artificial Intelligence from the KULeuven, Belgium. From 2005 to 2010 he was a research assistant within the SISTA research group of the Department of Electrical Engineering (ESAT), KU Leuven, Belgium. In May 2010 he received his Ph.D. at the KULeuven in the faculty of Applied Sciences, department of Electrical Engineering. He currently is a post-doctoral researcher in the AdvISe research group, technology campus Geel, KULeuven. His main research interest are machine learning and biomedical signal processing. From 2001 he combines his research with teaching in the areas of electronics, signal processing and machine learning.

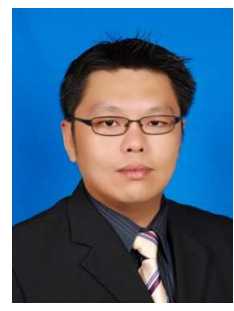

Ping Jack Soh (S'09) was born in Sabah, Malaysia. He received the Bachelor and Master degrees in Electrical Engineering (Telecommunication) from Universiti Teknologi Malaysia (UTM) in 2002 and 2005, respectively, and the Ph.D degree in Electrical Engineering from KU Leuven, Belgium in 2013.

$\mathrm{He}$ is currently a Senior Lecturer at the Advanced Communication Engineering (ACE) Center of Excellence, The School of Computer and Communication Engineering (SCCE), Universiti Malaysia Perlis (UniMAP). From 2002 to 2004, he was a Test Engineer in 
Venture Corp., working on new products' test definition for manufacturing purposes, both hardware and software. In 2005, he joined Motorola Solutions Penang, Malaysia, as an R\&D Engineer for Electrical Design. There, he worked on the hardware development of two-way radios, focusing on the characterization and testing of new radios' antennas and RF front-ends. From 2006, he joined SCCE-UniMAP as a Lecturer. He went on leave from UniMAP in 2009 to pursue his Ph.D and research attachment in the ESAT-TELEMIC Research Division, Department of Electrical Engineering, KU Leuven, Belgium. He was first a Research Assistant (2009-2013), and then a Postdoctoral Research Fellow (2013-2014) and is currently an External Research Affiliate. Within the context of his Ph.D, he was also involved in antenna design and characterization for AGFA Healthcare, Mortsel, Belgium. His research interest includes the design, development and modeling of flexible, textile, conformal and planar antennas, on-body communications, metamaterials and microwave measurements.

Dr. Soh was the recipient of the CST University Publication Award in 2011 and 2012, the IEEE Antennas and Propagation Society (AP-S) Doctoral Research Award in 2012, and the IEEE Microwave Theory and Techniques Society (MTT-S) Graduate Fellowship for Medical Applications in 2013. He was also the second place winner of the IEEE Presidents' Change the World Competition and IEEE MTT-S Video Competition, both in 2013.

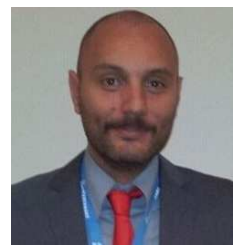

Giovanni Crupi (S'04-M'12-SM'13) was born in Lamezia Terme, Italy, in 1978. He received his M.Sc. in electronic engineering (with honors) and his Ph.D degrees from the University of Messina, Italy, in 2003 and in 2006, respectively.

Currently, he is Assistant Professor at the University of Messina, Italy, where he teaches "Microwave Electronics" and "Bioengineering". Since 2005, he has been a repeat Visiting Scientist with the University of Leuven (KU Leuven) and the Interuniversity Microelectronics Center (IMEC), Leuven, Belgium. His main research interests include small and large signal modeling of advanced microwave devices. He has authored or coauthored over 100 publications in international journals and conferences and two book chapters. He has coedit the book entitled "Microwave De-embedding: From Theory To Applications" (Academic Press, Oxford, UK, 2013).

Dr. Crupi is the Chair of the IEEE Microwave Theory and Techniques Society (MTT-S) Fellowship program. He serves as Associate Editor of the International Journal of Numerical Modelling: Electronic Networks, Devices and Fields. He also served as Technical Program Chair for the IEEE International Workshop on Integrated Nonlinear Microwave and Millimetre-wave Circuits (INMMiC), Leuven, Belgium, 2014.

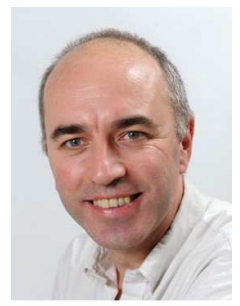

Guy A. E. Vandenbosch (F'13) received the M.S and Ph.D. degrees in Electrical Engineering from the Katholieke Universiteit Leuven, Leuven, Belgium, in 1985 and 1991, respectively.

Since 1993, he has been a Lecturer, and since 2005, a Full Professor at the same university. Guy Vandenbosch has teached or teaches courses on "Electromagnetic Waves", "Antennas", "Electromagnetic Compatibility", "Electrical Engineering, Electronics, and Electrical Energy", and "Digital Steer- and Measuring Techniques in Physics". His research interests are in the area of electromagnetic theory, computational electromagnetics, planar antennas and circuits, nano-electromagnetics, EM radiation, EMC, and bio-electromagnetics. His work has been published in ca. 210 papers in international journals and has lead to ca. 300 presentations at international conferences. From 2001 to 2007, he was the President of SITEL, the Belgian Society of Engineers in Telecommunication and Electronics. From 2008-2014, he was a member of the board of FITCE Belgium, the Belgian branch of the Federation of Telecommunications Engineers of the European Union. In the period 1999-2004, he was vice-chairman, and in the period 2005-2009 secretary of the IEEE Benelux Chapter on Antennas en Propagation.

Currently he holds the position of chairman of this Chapter. In the period 2002-2004 he was secretary of the IEEE Benelux Chapter on EMC. He currently is secretary of the Belgian National Committee for Radio-electricity (URSI), where he is also in charge of commission $\mathrm{E}$.

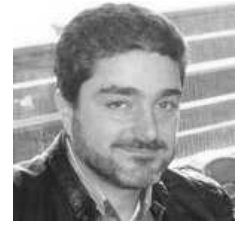

Calogero Pace (M'10) was born in Palermo, Italy, in 1965. He received the degree in Electronic Engineering in 1990 from the University of Palermo, and, in 1994, the Doctoral Degree in Electronic Engineering, Computer Science and Telecommunications from the same university.

Since 2002 he is associate professor of Electronics at University of Calabria, Italy. He has been the Coordinator of two Italy-Israel international project on the radiation hardness of electronic devices and systems for space applications. He is Associated Editor of the International Journal of Engineering and Industries and author of more than 110 scientific works, 59 of which published in refereed journals. Prof. Pace actual research interests are: development of innovative instrumentation and methodologies for the characterization of electronic devices; reliability issues of high mobility $(\mathrm{SiC}, \mathrm{GaN})$ power transistors; radiation effects on electronic devices and systems.

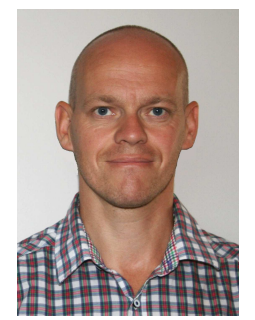

Paul Leroux (SM'10) was born in Eeklo, Belgium in 1975. He received the M.Sc. degree and Ph.D. degree in electronic engineering from the KU Leuven (University of Leuven), Belgium, in 1999 and 2004, respectively. From 1999 to 2004, he was a Teaching and Research Assistant within the MICAS research group of the KU Leuven Department of Electrical Engineering (ESAT).

Since 2009 he is an associate professor at KU Leuven and head of the Electrical Engineering (ESAT) Technology Cluster. He is a member of the Advance Integrated Sensing lab (AdvISe) and the MICAS research group. His current research activities focus on radiation tolerant IC design for nuclear fusion and high-energy physics applications, circuits for optical communication and instrumentation and electronics for ultra-wideband and microwave sensing systems. Prof. Leroux has (co)authored over 80 papers in international journals or conference proceedings.

In 2010, he received the SCK-CEN Prof. Roger Van Geen Award from the FWO and FNRS for his work on IC design for harsh radiation environments.

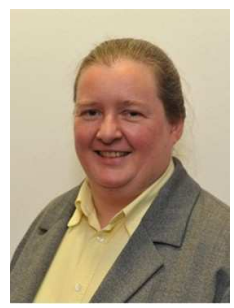

Dominique Schreurs (F'12) received the M.Sc. degree in electronic engineering and Ph.D. degree from the University of Leuven (KU Leuven), Belgium.

As post-doc fellow, she was visiting scientist with Agilent Technologies (USA), Eidgenössische Technische Hochschule Zürich (Switzerland), and the National Institute of Standards and Technology (USA). She is now full professor at KU Leuven. Her main research interests concern the nonlinear characterization and modelling of microwave and millimeter wave devices and circuits, as well as circuit and system design for telecommunications and biomedical applications.

Prof. D. Schreurs signed up as IEEE Student Member in 1990, and got elevated to Fellow in Jan. 2012. She serves on the IEEE MTT-S AdCom since 2009 , after election by the membership-at-large. She has served in various roles on the IEEE MTT-S AdCom, such as Chair of the MTT-S Education Committee (2012-2013). She is also past chair of the MTT-S Technical Committee on Microwave Measurements (MTT-11). Prof. D. Schreurs was Associate Editor for IEEE Microwave and Wireless Components Letters (2011-2013), and is now Editor of the IEEE Transactions on Microwave Theory and Techniques. She is also Distinguished Microwave Lecturer for the term 2012-2014. Beyond IEEE, Prof. D. Schreurs also serves on the Executive Committee of the ARFTG organization, presently as the Technical Chair. She was General Chair of the 2007 and 2012 Spring ARFTG Conferences. In 2002, she was one of the initiators and is now still co-organizer of the successful NVNA Users' Forum. Prof. D. Schreurs was also co-chair of the European Microwave Conference in 2008 and initiated the IEEE Women in Microwaves event at the European Microwave Week. She is also Associate Editor of the International Journal of Microwave and Wireless Technologies.

Prof. D. Schreurs is TPRC member of IMS and RWW and reviewer for many MTT-S (co-)sponsored conferences as well as for other IEEE journals and conferences. She has been regularly session chair at conferences, and acted as judge for student competitions. Prof. D. Schreurs is co-editor of four books, contributor to seven books, and (co-)author of over 100 journal papers and 350 contributions at international conferences. 